\title{
Increasing the gradient of energetic cost does not initiate adaptation in human
} walking

Surabhi N. Simha ${ }^{1}$, Jeremy D. Wong1,2, Jessica C. Selinger ${ }^{1,3}$, Sabrina J. Abram ${ }^{4}$, J. Maxwell Donelan $^{1^{*}}$

${ }^{1}$ Department of Biomedical Physiology and Kinesiology, Simon Fraser University, Burnaby, BC V5A 1S6, Canada

2Faculty of Kinesiology, University of Calgary, Calgary, AB T2N 1N4, Canada

Abstract

16 When in a new situation, the nervous system may benefit from adapting its control policy.

17 In determining whether or not to initiate this adaptation, the nervous system may rely on some features of the new situation. Here we tested whether one such feature is salient cost savings. We changed cost saliency by manipulating the gradient of participants' energetic cost landscape during walking. We hypothesized that steeper gradients would cause participants to spontaneously adapt their step frequency to lower costs. To manipulate the gradient, a mechatronic system applied controlled fore-aft forces to the waist of participants as a function of their step frequency as they walked on a treadmill. These forces increased the energetic cost of walking at high step frequencies and reduced it at low step frequencies. We successfully created three cost landscapes of increasing gradients, where the natural variability in participants' step frequency provided cost changes of 3.6\% (shallow), 7.2\% (intermediate) and 10.2\% (steep). Participants did not spontaneously initiate adaptation in response to any of the gradients. Using metronome-guided walkinga previously established protocol for eliciting initiation of adaptation-participants next

30 experienced a step frequency with a lower cost. Participants then adapted by $-1.41 \pm 0.81$

$31(\mathrm{p}=0.007)$ normalized units away from their originally preferred step frequency obtaining 32 cost savings of $4.80 \pm 3.12 \%$. That participants would adapt under some conditions, but not

33 in response to steeper cost gradients, suggests that the nervous system does not solely rely

34 on the gradient of energetic cost to initiate adaptation in novel situations. 


\section{Introduction}

37 We routinely perform movements in a variety of situations. This includes handling of

38 different-sized objects, walking on uneven terrain, or running with fatiguing muscles. Some

39 of these situations are familiar, and for these situations, our nervous system may have

40 already learned an optimal, or near-optimal, control policy (Izawa et al., 2008; Wolpert et

41 al., 2011; Wolpert and Flanagan, 2016). In the task of walking on a treadmill, for example,

42 people can rapidly select the step frequency that minimizes energetic cost for each new

43 walking speed (Pagliara et al., 2014; Snaterse et al., 2011). But in novel situations, the

44 nervous system hasn't had the experience to determine whether an existing policy remains

45 optimal, or if a new policy would be better (Wolpert et al., 2011; Wolpert and Flanagan,

46 2016). To determine this, the nervous system must adapt the existing policy and

47 experience the outcome (Sutton et al., 1992; Wolpert et al., 2011). This adaptation is

48 beneficial only when there is a new optimal solution, the presence of which the nervous

49 system does not know in advance. If the old policy remains the optimal policy, then the act

50 of adapting to new policies is itself sub-optimal-the nervous system would benefit most

51 by exploiting its existing control policy (Sutton et al., 2017). In this paper, we aim to

52 identify a feature of novel situations that cues the human nervous system to initiate

53 adaptation of its control policy.

55 Our nervous systems do not always initiate adaptation in novel situations. In reaching

56 experiments, people typically initiate adaptation when presented with a force-field that

57 creates a novel relationship between cost and control policy (Shadmehr and Mussa-Lvaldi,

58 1994; Wolpert et al., 2011). However, when this is followed by another force-field that

59 creates a different novel relationship, the nervous system reverts to erroneously exploiting

60 its original control policy (Gupta and Ashe, 2007; Wolpert et al., 2011). Similar interference

61 to adaptation is also observed in studies that create novel situations using visuomotor

62 rotations or reversals (Krakauer et al., 2019). In walking tasks, exoskeletons designed to

63 improve walking economy can underperform partly because people are unable to adapt

64 their gait to take full advantage of the benefits that the exoskeleton can offer (Jackson and

65 Collins, 2015; Wong et al., 2019; Zhang et al., 2017). In split-belt walking, people do not

66 adapt their step lengths back to baseline when the speeds of the two belts are changed 
67 gradually (Roemmich and Bastian, 2015). However, in all of these tasks, the nervous

68 system can and does adapt when certain modifications are made to the novel situations

69 (Krakauer et al., 2019; Selinger et al., 2015; Torres-Oviedo et al., 2011; Wolpert and

70 Flanagan, 2016; Zhang et al., 2017). This suggests that the nervous system relies on

71 particular features of the novel situations to determine if and when to initiate adaptation.

73 One potential feature used by the nervous system to initiate adaptation is salient cost

74 savings. Here we use cost savings to refer to an improvement in the nervous system's

75 objective function. This may be decreased energetic cost, increased stability, increased

76 accuracy, or some combination of these and other contributors to the objective function.

77 Saliency refers to how clear it is to the nervous system that cost savings can be gained, and

78 how it should adapt its control policy to gain the savings. As illustrated in Figure 1, saliency

79 depends on at least three factors. First, execution variability about the nominal policy-due

80 to either imperfect execution, purposeful exploration, or guidance by an external input-

81 allows the nervous system to experience a greater range of cost savings if they exist (Figure

82 1B). Second, measurement noise decreases the ability of the nervous system to discern the

83 presence of cost savings (Figure 1C). Third, for any given execution variability and

84 measurement noise, an increase in the gradient of the cost landscape increases the ability

85 of the nervous system to discern a cost savings (Figure 1D). If cost savings are not salient-

86 be it due to any combination of shallow cost gradient, high measurement noise, or low

87 execution variability-the nervous system may choose to exploit its current control policy

88 because whether it should adapt, and if so how it should adapt, is simply not clear. 
A) Probability $=0$ of detecting cost savings

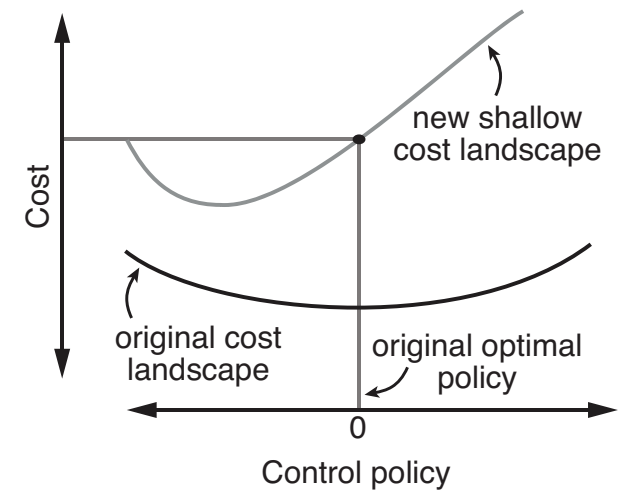

C) Low probability of detecting cost savings

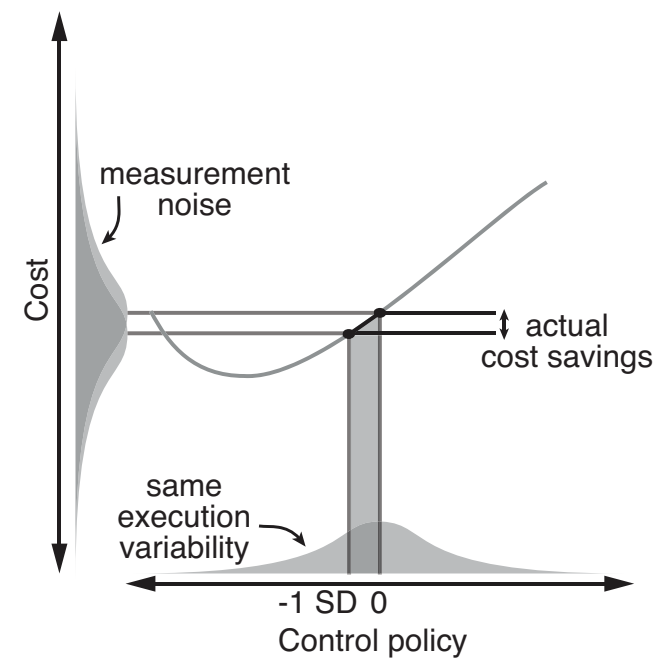

B) Probability $=1$ of detecting cost savings

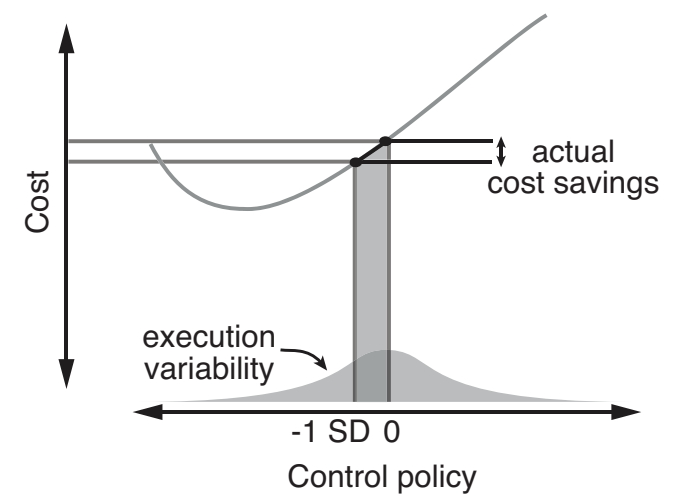

D) Higher probability of detecting cost savings

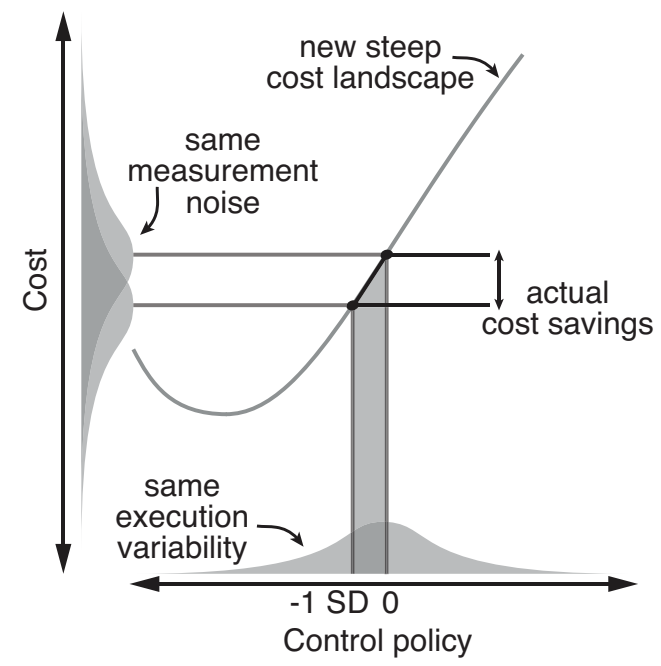

Figure 1: Conceptual representation of how the nervous system might detect cost savings from a cost

92 landscape. A) The nervous system is introduced to a novel situation where the relationship between the

93 control policy and cost has changed (black to grey curve) such that the original optimal policy is no longer

94 optimal. With exact execution and measurement, the nervous system cannot detect any cost savings in the

95 new landscape. B) Execution variability-illustrated by the horizontally aligned Gaussian distribution-

96 allows the nervous system to exactly experience the lower costs relative to the original policy, making the

97 energetic cost savings salient $\mathbf{C}$ ) The presence of measurement noise-illustrated by the two vertically-

98 aligned Gaussian distributions centered on the means of the two cost measurements-can reduce saliency by

99 reducing the probability that the nervous system can detect a cost savings. In this example, the cost

100 measurement means are close, and the cost measurement noise distributions are wide resulting in a low

101 probability that the nervous system will detect a cost savings for the given execution variability. D) An

102 increased gradient can increase the probability of detecting cost savings and thus increase the saliency of a

103 cost landscape for the same execution variability and measurement noise. 
105 Recent studies in walking support the premise that the nervous system relies on salient

106 cost savings to initiate adaptation. One of the primary real-time objectives of the nervous

107 system during walking is to minimize energetic cost (Abram et al., 2019; Selinger et al.,

108 2019, 2015; Simha et al., 2019). In one of our recent studies, we used robotic exoskeletons

109 to reshape the energetic cost landscape of treadmill walking. Here cost landscape refers to

110 the relationship between step frequency and metabolic energetic cost. We reshaped the

111 cost landscape to shift the optimal step frequency to step frequencies lower than normally

112 preferred. Upon their first experience with the new cost landscape, only some participants

113 spontaneously initiated adaptation to the new optimal step frequency. These spontaneous

114 initiators had greater step frequency variability than the non-spontaneous initiators who

115 persisted walking at the previous optimal step frequency. This suggests that the naturally

116 higher variability increased the saliency of the cost savings to the nervous system which

117 led to the initiation of adaptation. We were also able to prompt the non-spontaneous

118 initiators to initiate adaptation by providing them with experience with step frequencies

119 that resulted in a lower energetic cost. One interpretation of this result is that the

120 experience increased the saliency of the energetic cost savings for the nervous system

121 causing it to initiate further exploration. Counter to these findings, we did not find that

122 increased gait variability was sufficient to initiate adaptation in a subsequent study on over

123 ground walking (Wong et al., 2019). When compared to our treadmill studies, changes in

124 cost in this over ground study were due not only to changes in step frequency, but also

125 speed and terrain. We suspect that the nervous system did not initiate adaptation within

126 the duration of this over ground experiment because the added dimensionality increased

127 the complexity of the credit assignment problem making it difficult for the nervous system

128 to determine which energetic changes could be attributed to its control, and which were

129 due to the differences in terrain.

130

131 In the present study, we aimed to test whether the saliency of energetic cost savings is a

132 feature that the nervous system uses to initiate adaptation in human walking. To

133 accomplish this, rather than manipulate measurement noise or movement variability, we

134 changed saliency by manipulating the gradient of the energetic cost landscape. We

135 manipulated the gradient using a mechatronic system that applied controlled fore-aft 
136 forces to the waist of participants as they walked on a treadmill. These applied forces were

137 a function of participants' step frequency and acted to increase energetic cost at high step

138 frequencies and reduce it at low step frequencies. By making the forces a function of only

139 step frequency and keeping the walking speed constant, we aimed to only affect the

140 gradient of the step frequency cost landscape, indirectly signaling to the nervous system

141 how it should adapt its control policy to obtain cost savings. We increased the gradient of

142 the cost landscape about participants' originally preferred step frequency by increasing the

143 magnitude of force change that the system provided for a given change in step frequency.

144 We hypothesized that increasing the gradient of the cost landscape will cause participants

145 to spontaneously initiate adaptation of their step frequency.

\section{Methods}

\section{Experimental design:}

149 We manipulated cost landscapes using our recently developed mechatronic system (Figure

150 2A). We describe this system in detail in our earlier paper (Simha et al., 2019). Briefly, it

151 manipulates a participant's original cost landscape by applying fore-aft forces to their waist

152 while they walk on a treadmill. The controller specifies the forces as a function of the

153 participant's step frequency. Backward forces increase the energetic cost associated with

154 the executed step frequency, relative to normal, while moderate forward forces decrease

155 the energetic cost (Gottschall and Kram, 2003). The system uses inertial measurement

156 units placed on participants' feet to detect ground contact events, and this signal is

157 processed by a real-time controller to determine the participants' executed step frequency,

158 defined as the inverse of the time elapsed between left and right foot ground contact

159 events. We provide the controller with a control function that defines the relationship it has

160 to maintain between the measured step frequency and the applied force. Based on this

161 control function and the measured step frequency, the controller commands the required

162 force for each new step to an actuator via a motor driver. The force applied by the actuator

163 is transmitted to the participants through long tensioned cables that are attached to a hip

164 belt, and we monitor that force using force transducers in-line with the front and back

165 cables. 
A)

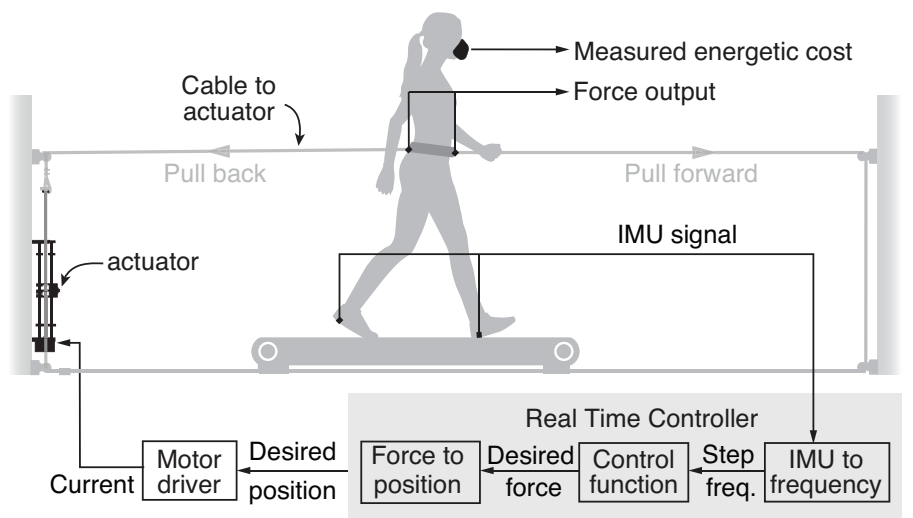

167

168

169

170

171

172

173

174

175

176

177

178

179

180

181

182

183

184

185

186

187 originally preferred step frequency $(0)$. steep - that created cost landscapes of three different gradients.
B)

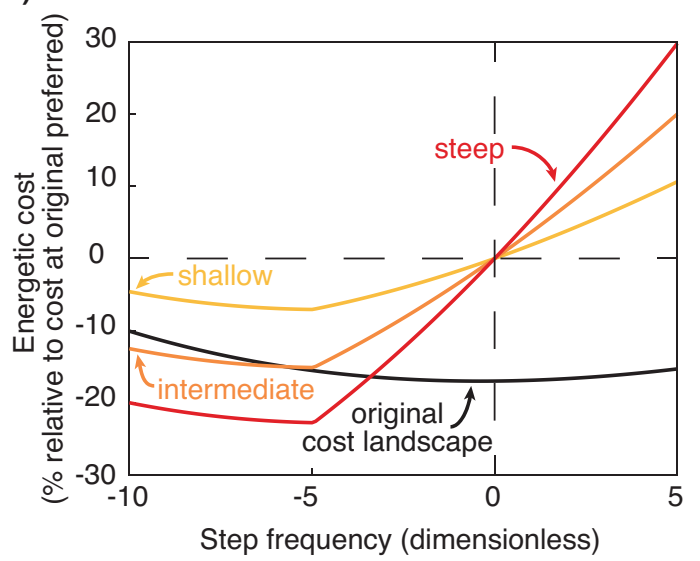

Figure 2: A) Participants walked in a mechatronic system that applied controlled fore-aft forces as a function of their walking step frequency. Backward forces provided an energetic penalty, raising the cost of walking relative to normal. Moderate forward forces provided an energetic reward, lowering energetic cost. B) Using simulations, we predicted that participants would experience cost landscapes with gradients of 1.4 (shallow), 2.8 (intermediate), and 4.2 (steep) percentage change in cost per unit change in step frequency, about their

We tested participants' behavior in cost landscapes of three different gradients (Fig 2B). Using data from literature, we can predict for an average participant the energetic cost associated with each step frequency when walking without any external force (Umberger and Martin, 2007), as well as the energetic cost of walking when a range of fore-aft forces are applied but at a fixed step frequency (Gottschall and Kram, 2003). We combined these relationships and used them to design three control functions-shallow, intermediate, and

$$
F_{\text {shallow }}=\left\{\begin{array}{l}
-0.07 \cdot s f-1.36, s f \leq-5 \\
-0.19 \cdot s f-1.98, \text { sf }>-5
\end{array}\right.
$$


189 Here, $s f$ is a normalized step frequency and is dimensionless. To perform this

190 normalization, we first measured the average step frequency originally preferred by each

191 participant during a baseline trial (c.f. Experimental Protocol), as well as the standard

192 deviation in step frequency about this average preferred step frequency. We then

193 calculated the normalized step frequency for each step in the subsequent trials by

194 subtracting the average originally preferred step frequency from each step's measured step

195 frequency and then dividing by the standard deviation about the originally preferred step

196 frequency. This normalization controls for the differences between participants in their

197 step frequency variability, which is normally one of the contributors to the saliency of cost

198 savings. It also forces measured step frequencies that are equal to the originally preferred

199 step frequency to evaluate to 0 . We normalized the forces applied to a participant by their

200 body weight. In equations 1-3, the intercepts, slopes, and the forces all have units of

201 percent body weight ( $s f$ is dimensionless). We designed these control functions to

202 generate new cost landscapes with cost gradients of 1.4, 2.8, and 4.2 about the originally

203 preferred step frequency. These gradients have units of percent change in energetic cost

204 for a unit change in normalized step frequency. For example, were a participant walking in

205 the intermediate gradient condition to choose a step frequency 1 normalized unit lower

206 than their originally preferred step frequency, the participant will experience a 2.8\%

207 reduction in energetic cost relative to what they experienced at the originally preferred

208 step frequency. For comparison, the cost landscape used in Selinger's study roughly

209 corresponds to the shallowest gradient we use here (Selinger et al., 2019, 2015). To

210 experience a cost gradient as steep as our steepest, one would have to walk at a step

211 frequency roughly 7.5\% higher than their preferred step frequency in their original cost

212 landscape (Umberger and Martin, 2007). Finally, we designed all the new cost landscapes

213 to have the same cost at the originally preferred step frequency. This helped ensure that

214 when we changed the cost landscape, participants only experienced the gradient change,

215 without experiencing any change in the average steady-state cost. Since different nervous

216 systems can respond differently to our control functions, each participant may not

217 experience exactly the cost landscape that we aimed to create. However, our prior results

218 show that, on average, we are able to accurately create our designed cost landscapes

219 (Simha et al., 2019). 


\section{Experimental protocol:}

222 We collected data from 11 participants (mean \pm SD; Age: $24 \pm 3$ years; Height: $167 \pm 11 \mathrm{~cm}$;

223 Mass: 68 $\pm 11 \mathrm{~kg}$; Gender: 5 females, 6 males). All participants were healthy and had no

224 known history of cardiopulmonary or gait impairments. The study protocol was approved

225 by the Simon Fraser University Research Ethics Board and all participants gave written

226 informed consent before participation. To determine the sample size necessary to evaluate

227 our hypothesis, we first performed pilot experiments and estimated that we can expect a

228 group standard deviation of 1.01 steps per minute. We then performed a power analysis for

229 a one-tailed Students' t-test to detect an average change of 1 normalized unit in step

230 frequency $(\alpha=0.05,1-\beta=0.90)$.

232 Each participant completed four periods of walking on the same day (Figure 3). Prior to the

233 beginning of these four experimental periods, all participants spent $\sim 10$ minutes

234 habituating to walking on our treadmill at a speed of $1.25 \mathrm{~m} \cdot \mathrm{s}^{-1}$. During this habituation, we

235 instructed them to walk with both short and long steps. They were not attached to the

236 mechatronic system. This was followed by the first period of the experiment where

237 participants walked for 9 minutes while attached to the mechatronic system. We used data

238 from this period to quantify the characteristics of their baseline walking step frequency.

239 During this time, the system controlled for a target applied force of $0 \mathrm{~N}$ (Figure 3A). We

240 calculated the average and standard deviation of their step frequency from the $6^{\text {th }}$ to $9^{\text {th }}$

241 minute to parameterize the step frequency in future trials. We refer to this average as the

242 originally preferred step frequency and the standard deviation as original step frequency

243 variability. 


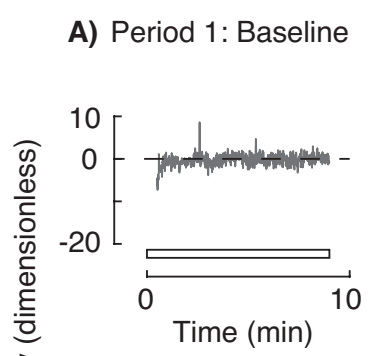

B) Period 2: Shallow and steep spontaneous C) Period 3: Intermediate spontaneous
and enforced experience
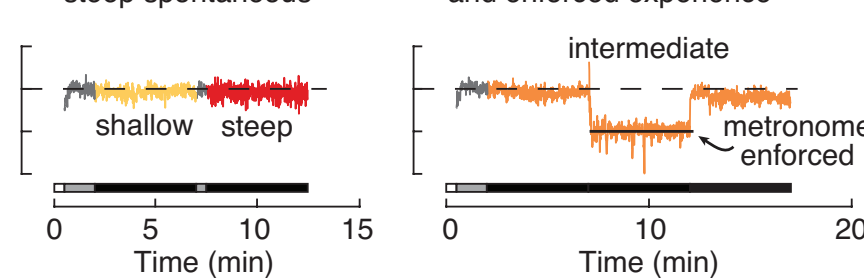

$\square \begin{aligned} & \text { No force applied } \\ & \text { (original landscape) }\end{aligned}$

Slow force change
to match force in new cost landscape

D) Period 4: Cost map

这 1

Figure 3: Step frequency measured from a representative participant during the different walking periods.

247 Each participant completed four periods of walking in a single day. A) First, they walked for 9 minutes as the

248 system controlled for a force of $0 \mathrm{~N}$ to be applied to their waist. We used this baseline period to estimate their average original preferred step frequency and original step frequency variability. B) Then participants walked for 5 minutes each in the shallow and steep gradients to test for spontaneous adaptation. C) In the third period, participants walked in an intermediate gradient. We used this condition to test for both spontaneous adaptation to an intermediate gradient and adaptation after enforced experience with a low cost. D) Finally, we measured the actual gradients experienced by participants in each the cost landscapes.

In the second period, we tested whether participants would spontaneously initiate adaptation in the shallow and steep gradients (Figure 3B). They experienced $0 \mathrm{~N}$ for the first $30 \mathrm{~s}$ to allow them to reach a steady-state step frequency (Pagliara et al., 2014). We programmed the system to ramp up the force over the next minute (minute 0.5 to 1.5 ) to 259 the force that would be applied at the participants' originally preferred step frequency in 260 the new cost landscapes. This ensured that participants were not perturbed by a sudden 261 change in force when the cost landscape changed. This force was held constant for $30 \mathrm{~s}$ 262 (minute 1.5 to 2; shallow pre-spontaneous). The controller then engaged the control 263 function for the shallow gradient, and participants walked at a self-selected step frequency 264 for 5 minutes (minute 2 to 7 ; shallow spontaneous). Then the controller switched to the 265 steep gradient. Once again, we ensured that participants were not perturbed during the 266 cost landscape transition by using a limit on the rate at which the force could change for 30 $267 \mathrm{~s}$ (minute 7 to 7.5). Participants then self-selected their step frequency for five minutes 
268 (minute 7.5 to 12.5; steep spontaneous). To avoid fatigue, we then provided a break of 5-10

269 minutes before beginning the third period. For each participant, we averaged their self-

270 selected step frequency over the last $30 \mathrm{~s}$ of walking in each gradient to determine their

271 spontaneous adaptation in that gradient (shallow spontaneous: minute 6.5 to 7; steep

272 spontaneous: minute 12 to 12.5$)$.

273

274 We used the third period to test for adaptation in an intermediate gradient (Figure 3C). The

275 first part of this period served as a sort of Goldilocks test in the event that the shallow and

276 steep gradients were both perceived as extreme by the nervous system (Kidd et al., 2012).

277 Similar to the second period, the force was ramped up in the first two minutes to prevent

278 perturbing forces. The controller then engaged the control function for the intermediate

279 gradient, and participants self-selected their step frequency for 5 minutes (minute 2 to 7;

280 intermediate spontaneous). One possible outcome of our experiment was that participants

281 would not spontaneously adapt in any of the gradients. With this outcome, we would not be

282 able to distinguish between the possibility that participants will adapt but not

283 spontaneously, and the possibility that participants won't adapt at all in our system with

284 our experimental paradigm. Therefore, the next part of this experimental period was to

285 verify whether adaptation was possible at all. Prior work has shown that experiencing a

286 lower cost in a new cost landscape is sufficient to cause the nervous system to initiate

287 adaptation (Selinger et al., 2019). Using this principle, we next required participants to

288 match their step frequency to an audio metronome that played a frequency -10 normalized

289 units away from their originally preferred step frequency. According to our designed cost

290 landscape, we expected this step frequency to provide a cost savings of $12.5 \%$ relative to

291 the cost at 0 . After five minutes of matching the metronome (minute 7 to 12; intermediate

292 metronome guided), the metronome was turned off and participants self-selected their step

293 frequency for another five minutes (minute 12 to 17; intermediate post-experience). Once

294 again, we averaged each participant's step frequency during the last $30 \mathrm{~s}$ of each condition

295 to determine their preferred step frequency in that condition (intermediate spontaneous:

296 minute 6.5 to 7; intermediate post-experience: minute 16.5 to 17 ). 
298 The purpose of the fourth period was to measure the actual energetic cost experienced by 299 the participants in each of the new cost landscapes (cost mapping; Figure 3D). During this 300 period, participants were also instrumented with a respiratory gas analysis system (Vmax

301 Encore Metabolic Cart, Viasys, Pennsylvania, USA). They spent the first six minutes 302 standing still while we measured their resting metabolic rate (minute 0 to 6 ). They then 303 started walking while the mechatronic system maintained a force of $0 \mathrm{~N}$ to allow them to 304 reach a steady-state gait (minute 6 to 7). Following this, participants walked at specific 305 walking conditions chosen to allow us to estimate the gradient about the originally 306 preferred step frequency in each of the cost landscapes, and also to estimate if the experience low period indeed allowed participants to experience a lower cost. Participants

308 walked in 10 conditions total: step frequencies of $0,-5$, and +5 in shallow, intermediate and 309 steep gradients, and -10 in only the intermediate gradient. We enforced this by instructing 310 participants to match an audio metronome that played these frequencies. We programmed

311 the controller to present these conditions in a random order to each participant, to prevent

312 any order effects on these metabolic energy measures. To determine energetic cost, we

313 measured the total volume of oxygen consumed and volume of carbon dioxide produced in

314 the last three minutes of each condition, and divided them by the duration over which they

315 were measured, to obtain the steady state average rates of oxygen consumption $\left(\dot{V}_{\mathrm{O}_{2}}\right)$ and

316 carbon dioxide production $\left(\dot{V}_{\mathrm{CO}_{2}}\right)$. We then estimated the metabolic rate using the following

317 equation (Adamczyk et al., 2006; Brockway, 1987; Weir, 1949):

$$
P_{\text {met,gross }}=\left(16.48 \frac{\mathrm{Ws}}{\mathrm{mlO}_{2}} \dot{V}_{\mathrm{O}_{2}}\right)+\left(4.48 \frac{\mathrm{Ws}}{\mathrm{ml} \mathrm{CO}_{2}} \dot{V}_{\mathrm{CO}_{2}}\right)
$$

319 We subtracted resting metabolic power for each participant and present net energetic cost

320 as the energy used per unit time normalized for the person's body mass. It has the units

$321 \mathrm{~W} \cdot \mathrm{kg}^{-1}$.

\section{Data analysis:}

324 We first determined the average gradients and metronome-guided cost that participants

325 experienced in each cost landscape. We used MATLAB's fitlm command to find the best

326 linear fit through the energetic costs at $-5,0,+5$ normalized step frequencies for each

327 participant, in each cost landscape. We define the cost landscape gradient for each 
328 participant as the slope of this fit. We also used a one-tailed paired Students' t-test to

329 determine whether the cost at a step frequency of -10 , where we held participants during

330 the intermediate metronome guided condition, is lower than the cost at a step frequency of

3310 in the intermediate gradient.

332

333 We evaluated whether participants spontaneously initiated adaptation in response to

334 steeper gradients. We first compared the preferred step frequency in the shallow gradient

335 with the originally preferred step frequency using a one-tailed Student's t-test. We found

336 that these values were indeed different, but we did not attribute this shift in preferred step

337 frequency to an adaptation in response to a new cost gradient (c.f. Results). To determine if

338 there was any additional changes in preferred step frequency in the steeper gradients, we

339 then compared the average step frequencies during the spontaneous adaptation periods in

340 the intermediate and steep gradient to the same period in the shallow gradient.

342 We also determined whether participants initiated adaptation after enforced experience

343 with a low cost. We used a one-tailed paired Students t-test to determine whether

344 participants' preferred step frequency after the intermediate metronome guided condition

345 was significantly lower than the average step frequency $30 \mathrm{~s}$ prior to the experience with

346 low cost. The step frequency in this 30s prior to the experience corresponds to the

347 spontaneous adaptation in the intermediate gradient, allowing us to determine whether

348 the metronome guided experience generated adaptation that did not occur spontaneously.

350 In the conditions where we observed adaptation, we characterized the rate of adaptation.

351 We did so because a preferred step frequency can arise from fast predictive processes that

352 can occur over a few seconds or optimization processes that can occur over tens or

353 hundreds of seconds (Pagliara et al., 2014). As described in the introduction, we are

354 interested in the slow process since it is indicative of the nervous system learning to adapt

355 its policy to a novel situation. We modelled each participant's adaptation of step frequency

356 over time as a two-process exponential. We first averaged the step frequency during the

357 last 30 s prior to the beginning of the condition of interest, and the step frequency during

358 the last $30 \mathrm{~s}$ of the condition. If these two averages were different, we normalized the step 
359 frequency data during that condition such that the average step frequency of the $30 \mathrm{~s}$ prior

360 to the condition evaluated to 0 , and the average of the last $30 \mathrm{~s}$ evaluated to 1 . We then

361 used least squares regression implemented through MATLAB's fitnlm function to model

362 these data as the sum of two exponentials (Pagliara et al., 2014). We used the time

363 constants from this model to estimate the duration of the optimization process.

\section{Results}

366 We were successful in creating cost landscapes of different gradients. We found that

367 participants on average experienced a shallow gradient of $0.07 \pm 0.03 \mathrm{~W} \cdot \mathrm{kg}^{-1}(\mathrm{mean} \pm \mathrm{SD})$, an

368 intermediate gradient of $0.14 \pm 0.03 \mathrm{~W} \cdot \mathrm{kg}^{-1}$, and a steep gradient of $0.20 \pm 0.04 \mathrm{~W} \cdot \mathrm{kg}^{-1}$

369 (Figure 4). This is calculated as the change in energetic cost per normalized unit of step

370 frequency. We use 1 standard deviation of participants' preferred step frequency in their

371 original cost landscape, to normalize the measured step frequency. This means that

372 participants experience the reported gradient through a variability of 0.5 standard

373 deviations higher and lower than their originally preferred step frequency. Thus, 1

374 standard deviation higher and lower than their originally preferred step frequency, which

375 accounts for $68 \%$ of their steps, would have allowed participants to experience a change in 376 energetic cost of 3.6\%, 7.2\%, and 10.2\% in the shallow, intermediate, and steep gradients,

377 respectively. We also found that when participants in the intermediate gradient were held -

37810 normalized step frequencies lower than their originally preferred step frequency, they

379 experienced an average cost savings of $8.1 \% \pm 9.1 \%$ relative to the cost at the originally

380 preferred step frequency $(\mathrm{p}=0.006)$. 
A) Shallow gradient

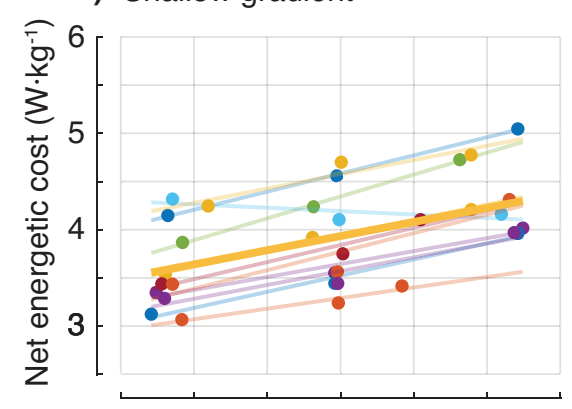

C) Steep gradient

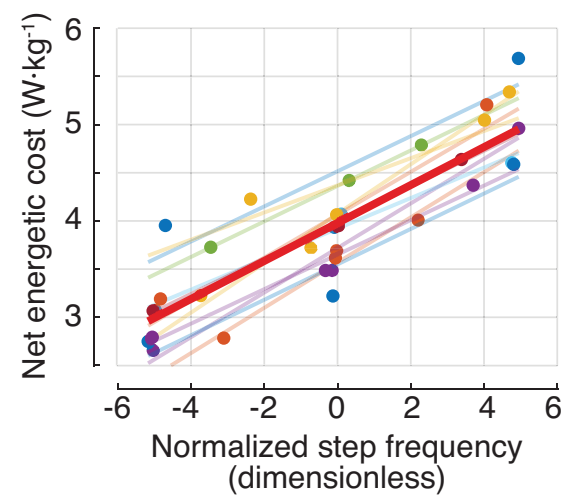

B) Intermediate gradient

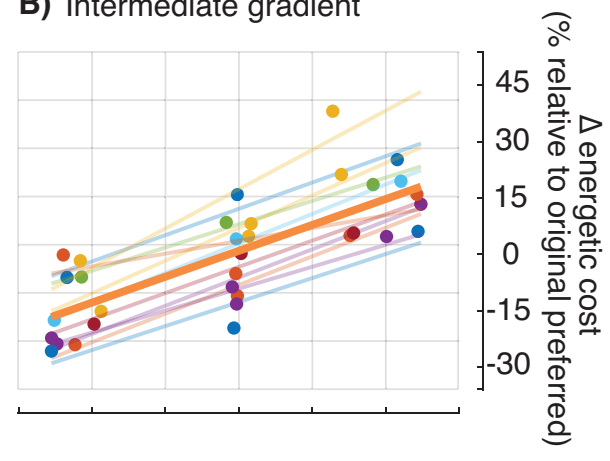

D) Average gradients

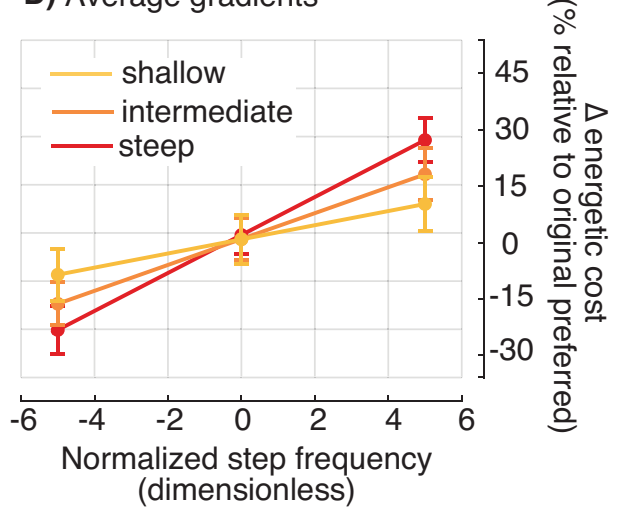

Figure 4: A) Shallow B) intermediate and C) steep gradients. Each filled circle represents one measurement from one participant. Light lines are linear fits to each participant's cost measurements. Data points and bestfit lines from a given participant is presented in a single colour. Thick lines are the average of these linear fits.

D) On average the gradients are increasing from shallow to steep. The filled circles represent the average cost measures at the commanded step frequencies, and the error bars represent the $95 \% \mathrm{CI}$ of the same.

388 Participants did not spontaneously initiate adaptation in response to steeper cost

389 gradients. In the second period, participants first experienced the shallow cost landscape, 390 and then the steep cost landscape. They walked freely at their self-selected step frequency

391 for $5 \mathrm{~min}$ in both cost landscapes. The average step frequency from the last 30 seconds of

392 the shallow period was lower than the original preferred step frequency $(-0.69 \pm 0.82$ vs. 0 ;

$393 \mathrm{p}=0.01$ ). However, this step frequency was indistinguishable from the average step

394 frequency preferred by participants during the $30 \mathrm{~s}$ prior to the beginning of the shallow

395 gradient (Figure 5: Shallow pre-spontaneous vs Shallow spontaneous; $-0.69 \pm 0.82$ vs. -

$3961.02 \pm 0.64 ; \mathrm{p}=0.33$ ). Therefore, we do not interpret this to be an initiation of adaptation

397 towards the optimal policy. When the system switched from the shallow landscape to the

398 steep landscape, participants still did not initiate adaptation, and preferred a step 
399 frequency (Figure 5: Steep spontaneous; $-0.76 \pm 0.99$ ) that was indistinguishable from that

400 preferred in the shallow cost landscape (Figure 5: Shallow spontaneous; $-0.69 \pm 0.82 ; \mathrm{p}=$

401 0.40). Our goldilocks test with the intermediate gradient also resulted in preferred step

402 frequencies that were indistinguishable from that preferred in the shallow landscape

403 (Figure 5: Shallow spontaneous vs Intermediate spontaneous; $-0.69 \pm 0.82$ vs. $-0.74 \pm 0.82 ; p$

$404=0.43)$.

405

406 Participants did, however, initiate adaptation after enforced experience with a lower cost.

407 We allowed participants to self-select their step frequency after matching a metronome

408 that held them at a step frequency that had a cost lower than the cost at 0 in the

409 intermediate cost landscape. On average, participants adapted by $-1.41 \pm 0.81$ towards the

410 new cost minimum (Figure 5: Intermediate post-experience). This adaptation was to step

411 frequencies significantly lower than that spontaneously preferred in the intermediate

412 gradient $(\mathrm{p}=0.007)$. It led to an average cost savings of $4.80 \pm 3.12 \%$ relative to the cost at 0 .

413 We found that the time course of the change in step frequency of most participants was

414 captured well with a two-process exponential model (RMSE=0.16 \pm 0.08 ; R-

415 squared $=0.36 \pm 0.21$ ). The time constant of the fast process was $4.4 \pm 2.5 \mathrm{~s}$ while that of the

416 slow process was $190.2 \pm 209$ s. We interpret the presence of this slow process as evidence

417 that the nervous system indeed initiated adaptation in response to the enforced experience

418 with a lower cost gait. 


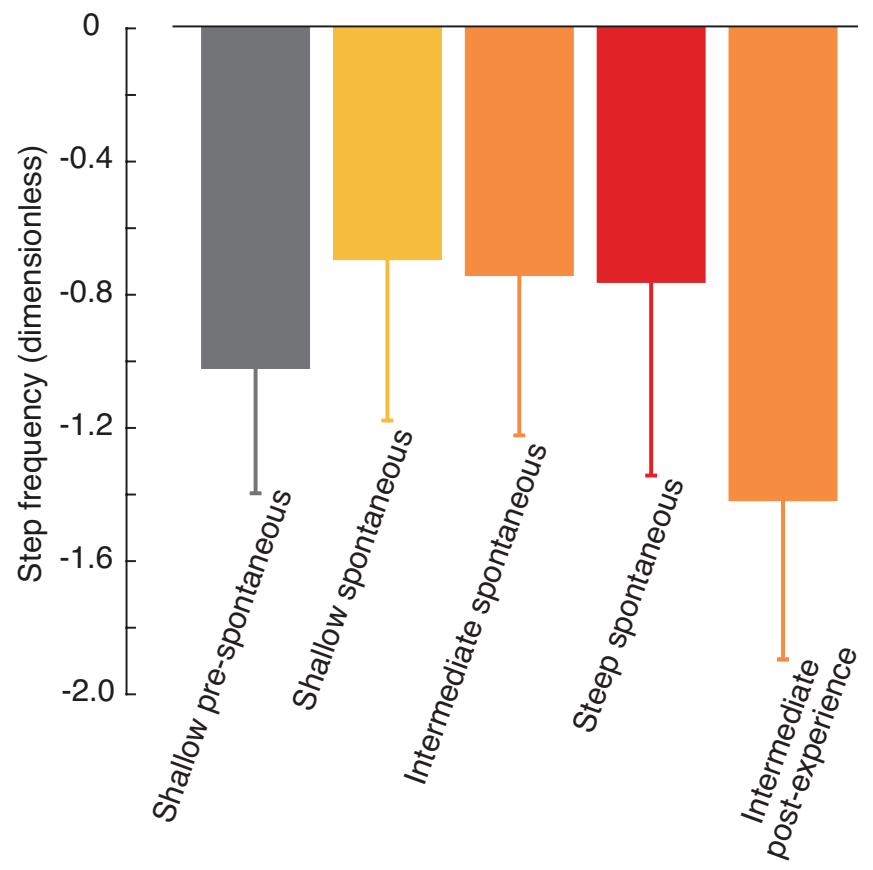

Figure 5: Average spontaneous adaptation in the shallow gradient was indistinguishable from participants' preferred step frequency just prior to the beginning of the shallow cost landscape (shallow pre-spontaneous vs shallow spontaneous). The spontaneous adaptation in all gradients were also indistinguishable from each

423 other after five minutes of walking (shallow, intermediate, steep spontaneous). However, after experience

424 with a lower cost in the intermediate gradient, participants preferred to walk at a significantly lower step

425 frequency (intermediate post-experience).

\section{Discussion}

427 Contrary to our hypothesis, steeper gradients did not lead to spontaneous initiation of 428 adaptation. This null finding is not because our methods were unsuccessful in creating 429 gradients of increasing steepness. We used our cost mapping trials to verify that the 430 participants did indeed experience three different gradients-the intermediate and steep 431 gradients were about 2-fold and 3-fold the shallow gradient, respectively. The lack of 432 initiation also does not appear to be a consequence of the rapid exposure to multiple 433 gradient conditions preventing the nervous system from attempting any adaptation. We 434 verified this by leveraging results from previous studies that found that adaptation can be 435 initiated by guiding the nervous system to experience a cost lower than the cost at the 436 originally preferred step frequency (Selinger et al., 2019). We did the same here and found 437 that participants could indeed initiate adaptation in the intermediate landscape after such 438 experience, despite the intermediate landscape being the third landscape experienced by 
participants. When considered together, these results suggest that either the nervous

440 system does not use salient cost savings to initiate adaptation, or that the cost savings were

441 not salient to the nervous system in our experiment.

443 For savings to be salient, the nervous system needs to both detect that cost savings can be

444 gained and determine how it should adapt its control policy to gain the savings. Depending

445 upon how the nervous system senses energetic cost, it may be challenging for the nervous

446 system to detect cost savings from the cost landscape gradient. For example, one possible

447 candidate sensory system for estimating energetic cost involves the ergoreceptors that are

448 sensitive to the slow build-up, or slow reduction, of muscle metabolic byproducts (Amann

449 et al., 2011; Iwamoto et al., 1985; Mitchell et al., 1983). This build-up creates a sensory

450 response that is an integration of the effect of many steps, rather than one that closely

451 follows the step-to-step changes in energetic cost. It will be more difficult for the nervous

452 system to detect a gradient in cost landscape from the step-to-step variability in energetic

453 cost when using this mechanism because integration has the effect of decreasing the sensed

454 gradient, perhaps even to zero if the build-up is particularly slow. This, or a similar

455 integrative sensing mechanism, may be why metronome-guided experience is effective at

456 initiating adaptation-the metronome holds participants at a lower cost for many steps

457 allowing time for integration. However, some participants in some conditions are able to

458 use the step-to-step variability in energetic cost to spontaneously initiate adaptation

459 (Selinger et al., 2019). This suggests that if a slow sensing system does indeed play a role in

460 estimating energetic cost, it is not the only contributing system.

462 Another possibility for the lack of initiation of adaptation is that the gradients allowed

463 participants' nervous systems to sense the presence of cost savings but not how to adapt

464 their control policy to obtain those savings. That is, the nervous system has difficulty with

465 credit assignment in our experiment (Guerguiev et al., 2020). We manipulated the cost

466 gradient associated with only one gait parameter-step frequency-to allow the nervous

467 system to detect an increase in cost savings and detect the gait parameter to adapt to

468 obtain those savings. But when walking in our system, we suspect that it is not clear to

469 most participants that the backward force depends on any aspect of their gait, including 
470 their step frequency. It appears to be challenging for the nervous system to identify salient

471 cost savings using the structure of natural variability in gait to determine the gradient of a

472 cost landscape-a finding consistent with our earlier experiment studying adaptation in

473 over ground walking (Wong et al., 2019). Metronome-guided experience of step

474 frequencies with lower cost may provide the nervous system with an explicit association

475 between the cost savings and the changes to control policy that provide those cost savings.

476 Similarly, reaching experiments have found that presenting participants with multiple

477 different force-fields interferes with learning, but that such interference can be overcome

478 with certain contextual cues such as follow through movements or cues that associate a

479 change in the optimal control policy with another change such as spatial location of

480 movement (Howard et al., 2015, 2013). Differences in contextual clues might explain why it

481 was easier for the nervous system to identify that there was a relationship between step

482 frequency and the changes to knee torque for some participants in our previous

483 experiment than with step frequency and torso forces in the present experiment (Selinger

484 et al., 2019). This interpretation is consistent with recent study in visuomotor adaptation

485 that found that implicit and explicit learning work together to improve adaptation

486 (Miyamoto et al., 2020).

488 While we designed our custom-built equipment and our protocol to meet the requirement

489 for energetic cost saliency, our experiment nevertheless had limitations. Towards this

490 requirement, the maximum cost savings that participants experienced from their

491 variability in step frequency, relative to the cost at their originally preferred step

492 frequency, was 5.1\% in the steep gradient. In contrast, participants experienced cost

493 savings of $8.2 \%$ during their metronome-guided lower cost experience in the intermediate

494 gradient. This suggests that even the steep gradient may not have allowed participants to

495 experience a large enough cost savings. However, we suspect this is not the case because in

496 our previous study with the shallow gradient, participants initiated adaptation after

497 experiencing cost savings of only 3.5\% through similar metronome-guided walking (Simha

498 et al., 2019). This earlier cost savings was smaller than that experienced by our current

499 participants in the steep gradient condition suggesting that the currently experienced cost 
500 savings, at least in the steep gradient condition, were sufficiently large for the nervous

501 system to detect.

502

503 A second limitation is that our experimental design resulted in participants preferring step

504 frequencies slightly lower than the original preferred step frequencies in all gradient

505 conditions (Figure 5). We do not interpret these shifts as evidence of the initiation of

506 energetic cost optimization in response to new cost landscapes. Our rationale is that

507 participants were already walking at shifted step frequency during the $30 \mathrm{~s}$ prior to the

508 beginning of each new cost landscape (shallow: $-1.02 \pm 0.64$, intermediate: $-0.74 \pm 0.82$,

509 steep: $-0.62 \pm 0.54)$. Why is step frequency shifted lower than the baseline measures both

510 before and during the experience with the new cost landscapes? One possible explanation

511 is that we may not have provided a long enough baseline period for participants to settle

512 into their preferred step frequency. However, others have found that two minutes of

513 walking is sufficient for stride frequency to reach steady state-we provided 9 minutes

514 (Van de Putte et al., 2006; Wall and Charteris, 1981). A second possible explanation for the

515 presence of these shifts may be the net backward force that participants experienced both

516 immediately before and during the cost landscape, but not during the baseline phase when

517 the net force was zero. Our system slowly ramped up the backward force to that which

518 participants would experience in the new cost landscapes at their originally preferred step

519 frequency. The force was then held constant for $30 \mathrm{~s}$ before the controller switched to the

520 new cost landscapes, and our step frequency estimate prior to the beginning of the new

521 cost landscape is from this constant-force period. However, concerned about the possible

522 role of net backward force on step frequency, we performed pilot experiments prior to our

523 reported experiments and found no relationship. In support of our pilot results, a recent

524 study also found that backward forces do not have an effect on stride period (Dewolf et al.,

525 2020). Furthermore, walking uphill, which is biomechanically similar to experiencing a net

526 backward force, also results in step frequencies that are not significantly different from

527 walking on level (Ortega and Farley, 2015). Further research will be required to

528 understand why we observed this consistent shift in step frequency. 
530 After metronome-guided experience, our participants did not converge on the energy

531 minimal step frequency. To determine the location of the cost minimum, and the magnitude

532 of cost savings obtainable at the minimum, we fit a quadratic relationship to the costs

533 measured in the intermediate gradient condition during cost mapping. From this

534 relationship, we estimate that, on average, participants could have obtained a cost savings

535 of $10.8 \%$ if they had shifted their step frequency -6.1 normalized units away from their

536 originally preferred step frequency. Yet we found that participants only adapted their step

537 frequency by $-1.4 \pm 0.8$ to obtain a cost savings of only $4.83 \pm 3.61 \%$. This might suggest to

538 some that energetic cost savings do not play a role in the adaptation of step frequency in

539 the cost landscapes we used. We suspect that this is not the case since participants did

540 adapt after experience with a lower energetic cost. A candidate explanation is that the

541 nervous system seeks to minimize an objective function that is a combination of energetic

542 cost, stability, accuracy and other contributors (Abram et al., 2019). The minimum of this

543 combined cost function may coincide with the final preferred step frequency and not with

544 the energetic cost minimum.

545

546 In conclusion, the nervous system does not solely rely on the gradient of energetic cost to

547 initiate adaptation in novel situations. As we and others have previously found, explicit

548 experience with more optimal movements can assist with the initiation of adaptation. A

549 better understanding of the interplay between implicit and explicit experience for the

550 nervous system to initiate adaptation when the saliency of cost savings is not apparent may

551 help improve rehabilitation for those recovering from injuries, help coaches speed up

552 training with new techniques, or aid scientists looking to study adaptation in complex

553 novel environments.

554

555

\section{References}

557 Abram, S.J., Selinger, J.C., Donelan, J.M., 2019. Energy optimization is a major objective in

558 the real-time control of step width in human walking. J. Biomech. 91, 85-91. https://doi.org/10.1016/j.jbiomech.2019.05.010 
560 Adamczyk, P.G., Collins, S.H., Kuo, A.D., 2006. The advantages of a rolling foot in human walking. J. Exp. Biol. 209, 3953-3963. https://doi.org/10.1242/jeb.02455

562 Amann, M., Blain, G.M., Proctor, L.T., Sebranek, J.J., Pegelow, D.F., Dempsey, J.A., 2011.

563

564

565

566

567

568

569

570

571

572

573

574

575

576

577

578

579

580

581

582

583

584

585

586

587

588

589

590 Implications of group III and IV muscle afferents for high-intensity endurance exercise performance in humans. J. Physiol. 589, 5299-5309. https://doi.org/10.1113/jphysiol.2011.213769

Brockway, J.M., 1987. Derivation of formulae used to calculate energy expenditure in man. Hum. Nutr. Clin. Nutr. 41, 463-471.

Dewolf, A.H., Ivanenko, Y.P., Mesquita, R.M., Lacquaniti, F., Willems, P.A., 2020. Neuromechanical adjustments when walking with an aiding or hindering horizontal force. Eur. J. Appl. Physiol. 120, 91-106. https://doi.org/10.1007/s00421-01904251-1

Gottschall, J.S., Kram, R., 2003. Energy cost and muscular activity required for propulsion during walking. J. Appl. Physiol. 94, 1766-1772. https://doi.org/10.1152/japplphysiol.01190.2004

Guerguiev, J., Kording, K.P., Richards, B.A., 2020. Spike-based causal inference for weight alignment. ArXiv191001689 Cs Q-Bio.

Gupta, R., Ashe, J., 2007. Motor Force Field Learning Influences Visual Processing of Target Motion. J. Neurophysiol. 97, 738-745.

Howard, I.S., Wolpert, D.M., Franklin, D.W., 2015. The Value of the Follow-Through Derives from Motor Learning Depending on Future Actions. Curr. Biol. 25, 397-401. https://doi.org/10.1016/j.cub.2014.12.037

Howard, I.S., Wolpert, D.M., Franklin, D.W., 2013. The effect of contextual cues on the encoding of motor memories. J. Neurophysiol. 109, 2632-2644. https://doi.org/10.1152/jn.00773.2012

Iwamoto, G.A., Waldrop, T.G., Kaufman, M.P., Botterman, B.R., Rybicki, K.J., Mitchell, J.H., 1985. Pressor reflex evoked by muscular contraction: contributions by neuraxis levels. J. Appl. Physiol. 59, 459-467. https://doi.org/10.1152/jappl.1985.59.2.459

Izawa, J., Rane, T., Donchin, O., Shadmehr, R., 2008. Motor Adaptation as a Process of Reoptimization. J. Neurosci. 28, 2883-2891. https://doi.org/10.1523/JNEUROSCI.5359-07.2008 
591 Jackson, R.W., Collins, S.H., 2015. An experimental comparison of the relative benefits of

592

593

594

595

596

597

598

599

600

601

602

603

604

605

606

607

608

609

610

611

612

613

614

615

616

617

618

619

620

work and torque assistance in ankle exoskeletons. J. Appl. Physiol. 541-557. https://doi.org/10.1152/japplphysiol.01133.2014

Kidd, C., Piantadosi, S.T., Aslin, R.N., 2012. The Goldilocks Effect: Human Infants Allocate Attention to Visual Sequences That Are Neither Too Simple Nor Too Complex. PLoS ONE 7. https://doi.org/10.1371/journal.pone.0036399

Krakauer, J.W., Hadjiosif, A.M., Xu, J., Wong, A.L., Haith, A.M., 2019. Motor Learning. Compr. Physiol. 9, 613-663.

Mitchell, J.H., Kaufman, M.P., Iwamoto, G.A., 1983. The Exercise Pressor Reflex: Its Cardiovascular Effects, Afferent Mechanisms, and Central Pathways. Annu. Rev. Physiol. 45, 229-242. https://doi.org/10.1146/annurev.ph.45.030183.001305

Miyamoto, Y.R., Wang, S., Smith, M.A., 2020. Implicit adaptation compensates for erratic explicit strategy in human motor learning. Nat. Neurosci. https://doi.org/10.1038/s41593-020-0600-3

Ortega, J.D., Farley, C.T., 2015. Effects of aging on mechanical efficiency and muscle activation during level and uphill walking. J Electromyogr Kinesiol 25, 193-198. https://doi.org/10.1109/TMI.2012.2196707.Separate

Pagliara, R., Snaterse, M., Donelan, J.M., 2014. Fast and slow processes underlie the selection of both step frequency and walking speed. J. Exp. Biol. 2939-2946. https://doi.org/10.1242/jeb.105270

Roemmich, R.T., Bastian, A.J., 2015. Two ways to save a newly learned motor pattern. J. Neurophysiol. 113, 3519-3530. https://doi.org/10.1152/jn.00965.2014

Selinger, J.C., O’Connor, S.M., Wong, J.D., Donelan, J.M., 2015. Humans Can Continuously Optimize Energetic Cost during Walking. Curr. Biol. 25, 2452-2456. https://doi.org/10.1016/j.cub.2015.08.016

Selinger, J.C., Wong, J.D., Simha, S.N., Donelan, J.M., 2019. How humans initiate energy optimization and converge on their optimal gaits. https://doi.org/10.1242/jeb.198234

Shadmehr, R., Mussa-Lvaldi, F.A., 1994. Adaptive Representation of Dynamics during Learning of a Motor Task. J. Neurosci. 74. 
621 Simha, S.N., Wong, J.D., Selinger, J.C., Donelan, J.M., 2019. A Mechatronic System for Studying

622

623

624

625

626

627

628

629

630

631

632

633

634

635

636

637

638

639

640

641

642

643

644

645

646

647

648

649

650

Energy Optimization During Walking. IEEE Trans. Neural Syst. Rehabil. Eng. 27, 1416-1425. https://doi.org/10.1109/TNSRE.2019.2917424

Snaterse, M., Ton, R., Kuo, A.D., Donelan, J.M., 2011. Distinct fast and slow processes contribute to the selection of preferred step frequency during human walking. J. Appl. Physiol. Bethesda Md 1985 110, 1682-1690. https://doi.org/10.1152/japplphysiol.00536.2010

Sutton, R.S., Barto, A.G., Book, A.B., 2017. Reinforcement Learning: An Introduction.

Sutton, R.S., Barto, A.G., Williams, R.J., 1992. Reinforcement Learning is Direct Adaptive Optimal Control.

Torres-Oviedo, G., Vasudevan, E., Malone, L., Bastian, A.J., 2011. Locomotor adaptation, in: Progress in Brain Research. Elsevier, pp. 65-74. https://doi.org/10.1016/B978-0444-53752-2.00013-8

Umberger, B.R., Martin, P.E., 2007. Mechanical power and efficiency of level walking with different stride rates. J. Exp. Biol. 210, 3255-65. https://doi.org/10.1242/jeb.000950

Van de Putte, M., Hagemeister, N., St-Onge, N., Parent, G., de Guise, J. a, 2006. Habituation to treadmill walking. Biomed. Mater. Eng. 16, 43-52.

Wall, J.C., Charteris, J., 1981. A kinematic study of long-term habituation to treadmill walking. Ergonomics 24, 531-542.

Weir, J.B. de V., 1949. New methods for calculating metabolic rate with special reference to protein metabolism. J. Physiol. 109, 1-9. https://doi.org/10.1113/jphysiol.1949.sp004363

Wolpert, D.M., Diedrichsen, J., Randall Flanagan, J., 2011. Principles of sensorimotor learning. Nat. Rev. Neurosci. 12, 739-751. https://doi.org/10.1038/nrn3112

Wolpert, D.M., Flanagan, J.R., 2016. Computations underlying sensorimotor learning. Curr. Opin. Neurobiol. 37, 7-11. https://doi.org/10.1016/j.conb.2015.12.003

Wong, J.D., Selinger, J.C., Donelan, J.M., 2019. Is natural variability in gait sufficient to initiate spontaneous energy optimization in human walking? J Neurophysiol 121, 1848-1855. https://doi.org/10.1152/jn.00417.2018.-In 
651 Zhang, J., Fiers, P., Witte, K.A., Jackson, R.W., Poggensee, K.L., Atkeson, C.G., Collins, S.H., 652 2017. Human-in-the-loop optimization of exoskeleton assistance during walking.

653 Science 356, 1280 LP - 1284. https://doi.org/10.1126/science.aal5054

654 